\title{
Mass segregation of different populations inside the cluster NGC $6101^{\star, \star \star}$
}

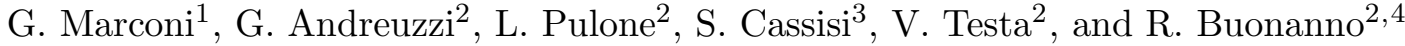 \\ 1 European Southern Observatory, Casilla 19001, Santiago, Chile \\ 2 Osservatorio Astronomico di Roma, via Frascati 33, 00040 Monteporzio Catone, Italy \\ 3 Osservatorio Astronomico di Collurania, via M. Maggini, 64100 Teramo, Italy \\ 4 Dipartimento di Fisica, Università di Roma "Tor Vergata", 00173 Roma, Italy
}

Received 14 August 2001 / Accepted 2 October 2001

\begin{abstract}
We have used ESO telescopes at La Silla and the Hubble Space Telescope (HST) in order to obtain accurate $B, V, I$ CCD photometry for the stars located within $200^{\prime \prime}\left(\simeq 2\right.$ half-mass radii, $\left.r_{\mathrm{h}}=1.71^{\prime}\right)$ from the center of the cluster NGC 6101. Color-Magnitude Diagrams (CMDs) extending from the red-giant tip to about 5 magnitudes below the main-sequence turnoff MSTO $(V=20.05 \pm 0.05)$ have been constructed. The following results have been obtained from the analysis of the CMDs: a) The overall morphology of the main branches confirms previous results from the literature, in particular the existence of a sizeable population of 73 "blue stragglers" (BSS), which had been already partly detected (27). They are considerably more concentrated than either the subgiant branch (SGB) or the main sequence (MS) stars, and have the same spatial distribution as the horizontal branch (HB) stars (84\% probability from K-S test). An hypothesis on the possible BSS progeny is also presented. b) The HB is narrow and the bulk of stars is blue, as expected for a typical metal-poor globular cluster. c) The derived magnitudes for the HB and the MSTO, $V_{\mathrm{ZAHB}}=16.59 \pm 0.10, V_{\mathrm{TO}}=20.05 \pm 0.05$, coupled with the values $E(B-V)=0.1,[\mathrm{Fe} / \mathrm{H}]=-1.80, Y=0.23$ yield a distance modulus $(m-M)_{V}=16.23$ and an age similar to other "old" metal-poor globular clusters. In particular, from the comparison with theoretical isochrones, we derive for this cluster an age of 13 Gyrs. d) By using the large statistical sample of Red Giant Branch (RGB) stars, we detected with high accuracy the position of the bump in the RGB luminosity function. This observational feature has been compared with theoretical prescriptions, yielding a good agreement within the current theoretical and observational uncertainties.
\end{abstract}

Key words. globular clusters: individual (NGC 6101) - stars: fundamental parameters - Hertzsprung-Russell diagram - stars: abundances - stars: horizontal branch

\section{Introduction}

NGC 6101 is a "typical" metal-poor galactic globular cluster located at $\alpha=16^{\mathrm{h}} 20.0^{\mathrm{m}}, \delta=-72^{\circ} 05^{\prime},\left(l=318^{\circ}\right.$, $\left.b=-16^{\circ}\right)$ with a low central concentration $\left(\log \rho_{0}=1.57\right.$, Djorgovski 1993).

A photographic study of the cluster was done by Alcaino (1974); there are only two important photometric studies based on CCD data: Sarajedini \& Da Costa (1991, SDC) and Rosenberg et al. (2000). The most

Send offprint requests to: G. Marconi,

e-mail: gmarconi@eso.org

* Based on observations collected at the European Southern Observatory, La Silla Chile and on HST observations.

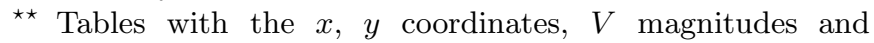
$(V-I),(B-V)$ colors (for both ground and HST-data), are only available in electronic form at the CDS via anonymous ftp cdsarc.u-strasbg.fr (130.79.128.5) or via http://cdsweb.u-strasbg.fr/cgi-bin/qcat?J/A+A/380/478 complete analysis was performed by SDC by using $B$ and $V$ observations of 4 fields in the external regions of the cluster. The main results of SDC are: (1) NGC 6101 is a "normal" metal-poor globular cluster of an age similar to other galactic globular clusters with similar metal abundances; (2) it contains a conspicuous fraction of BSS (27) with properties similar to the BSS of other globular clusters; in particular, from the analysis of radial distributions, they found that the BSS are more concentrated than the SGB stars.

We have obtained new ground-based and $\operatorname{HST} B, V$, $I$ high-quality photometry of $400 \operatorname{arcsec}^{2}$ centered on the cluster. The new data-set made it possible to re-analyse the stellar content inside $\simeq 2 r_{\mathrm{h}}$. For the sake of comparison, both CCDs studies quoted above have been used.

In Sect. 2 we describe the observations and the procedures applied in order to reduce and calibrate the data. Section 3 shows the $(V, B-V)$ and $(V, V-I)$ CMDs. 


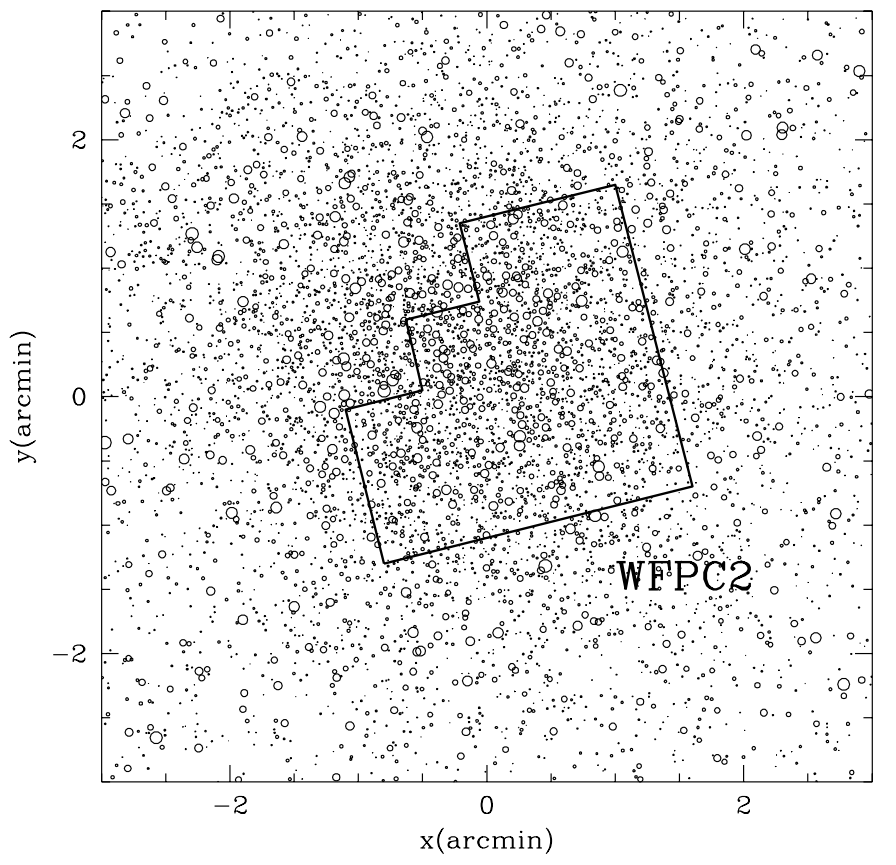

Fig. 1. Plot of the location of the HST-field inside the groundfield. The dimensions of the circle (representing the stars belonging to ground sample) depend on their magnitude. The magnitude limit of the stars reported in the figure is $V=21$.

The luminosity functions (LFs) and the radial distributions of the stars are discussed in Sects. 4 and 5. In Sect. 6 age, distance modulus and metallicity are derived from the comparison with theoretical models. The results are summarized in Sect. 7 .

\section{Observations and data reduction}

\subsection{Observations}

The ground-based $B, V, I$ images have been obtained at ESO/La Silla with the 1.54 Danish telescope and the Direct CCD camera. A large number of CCD frames of a field centered on NGC 6101 were obtained under good seeing conditions, during the observing run of July 1995. The CCD (a Tektronik 1024 with scale $0.37^{\prime \prime} /$ pixel and field of view of $400 \operatorname{arcsec}^{2}$ ) sampled very well the seeing profile which varies from $0.75^{\prime \prime}$ to $0.95^{\prime \prime}$. Standard stars for calibration were taken every night from Landolt's regions (Landolt 1992).

In order to carefully check the crowding effect in the most central region, deep exposures with HST + WFPC2 (with the filters $F 555 \mathrm{~W}$ and $F 814 \mathrm{~W}$ ) were obtained for the center of the cluster within the GO 6625 program (Cycle 6).

The location of the HST field, overlapped onto the field covered by the ground-based observations, is shown in Fig. 1. A journal of observations is given in Table 1, where the columns contain respectively: the filter used, the number of exposures and the exposure times in seconds for both data-sets.
Table 1. Journal of observations.

\begin{tabular}{c|ccc}
\hline & filter & $N$ & $t(\mathrm{~s})$ \\
\hline 1.54 Danish & $I$ & 2 & 10 \\
& & 1 & 30 \\
& & 2 & 60 \\
& & 1 & 120 \\
& & 2 & 240 \\
& & 2 & 10 \\
& & 1 & 30 \\
& & 2 & 60 \\
& & 3 & 300 \\
& & 2 & 60 \\
& & 1 & 180 \\
& & 3 & 600 \\
& & & \\
& & & 3 \\
& & & 3 \\
& & & \\
& & & 160 \\
& & 3 & 3 \\
& & 1 & 160 \\
& & 4 & 200 \\
& & 1 & 350 \\
\hline
\end{tabular}

We used the stars lying in the central region common to the HST and the Danish field, to carefully check the independent calibrations, and to make the data-sets uniform.

\subsection{Data reduction}

Corrections to the raw ground-data for cosmic rays, bias, dark and flat-fielding were made through the standard procedure. On the other hand we applied DAOPHOT-II (Stetson 1987, 1992) available on MIDAS for subsequent data reduction.

The first step of this procedure was the search of the stellar objects on the deepest $I$ image. The detected candidates were fitted in all $V, I$ and $B$ frames by using a PSF-profile and an average instrumental magnitude was derived for each object and color.

A total of 8413 objects have been detected in all the three bands down to $V \sim 22.5$. Internal errors were estimated as the rms frame-to-frame scatter of the instrumental magnitudes obtained for each star. The mean error in the interval $14 \leq V \leq 22$ turned out to be $\sigma_{B}=0.06$ $\sigma_{V}=0.05$ and $\sigma_{I}=0.04$.

Conversion from instrumental magnitudes to the Johnson standard system was performed by using primary calibrators (Landolt 1992), which covered a larger range in color than the cluster stars.

Corrections to the raw HST-data for bias, dark and flat-fielding were applied using the standard HST pipeline. Subsequent data reduction was made using MIDAS routines and the Romafot package for crowded fields (Buonanno \& Iannicola 1989).

First, a median filter was applied to each frame in order to remove cosmic rays from every single frame. Then we used the deepest $I$ image to search for stellar objects in each WFPC2 camera. 
All detected objects were fitted in all $V$ and $I$ frames using a PSF-profile modelled by a Moffat function, plus a numerical map of the residuals, to take into account the contribution of the stellar wings better. The identified candidates were then measured on each individual $V$ and $I$ frame, and an average instrumental magnitude was derived for each object and color.

The instrumental magnitudes were transformed into the standard Johnson system by using the Holtzman synthetic equations (Holtzman et al. 1995).

\section{Color-Magnitude Diagrams}

Figure 2 (right panel) shows the $V$ vs. $(V-I)$ CMD for 12012 stars detected in the HST sample.

Figure 2 (left panels) shows:

1. the $V$ vs. $(V-I)$ CMD for 9435 stars detected in the $V$ and $I$ filters in the ground-sample. The overimposed curve is the ridge line obtained (i.e. see details in Sect. 3.1) by fitting a gaussian to the distribution of $(V-I)$ values in each fixed magnitude $V$ intervals of the HST-CMD;

2. the $V$ vs. $B-V$ CMD for 8416 stars simultaneously detected in the $B$ and $V$ filters of the ground-sample. The overlapped line is the fiducial sequence $V$ vs. $(B-V)$ shown in Table 2 of SDC.

Because of the fainter magnitude level reached in the central regions when using HST ( $V \simeq 25.5)$, the number of objects present in the HST sample is larger than the number detected in the ground-based data, even if the spatial coverage is larger for the latter (i.e. $\simeq 7.7$ times in area).

In the three diagrams of Fig. 2, the richness of the samples allows the identification of all the evolutionary phases. In particular, a well populated main sequence, subgiant and giant branches, as well as the less represented blue horizontal branch, blue stragglers sequence and asymptotic giant branch (the last actually better seen in the ground based data of Fig. 2) are clearly visible. The overall morphology of the cluster (blue HB, steep RGB) confirms the metal-poor nature of NGC 6101, as already pointed out by Zinn \& West (1984, ZW), SDC and Rosenberg et al. (2000). In the following sections, we will carry out the analysis of each sequence, and we will estimate the fundamental quantities for this cluster, namely, reddening, distance, age and metallicity.

\subsection{Main Sequence and Red Giant Branch}

In Fig. 2 (right) we can appreciate a very narrow MS: the dispersion $\sigma(V-I)$, starts to grow remarkably $(\geq 0.037)$ about one magnitude below the MSTO: $V=20.05 \pm 0.05$, $(V-I) \simeq 0.69$. In particular, this value has been obtained by using the following procedure: first of all, we considered an interval one magnitude $V$ wide, around the most probable blue point of the MS in the CMD of Fig. 2 (right).
Table 2. RGB fiducial line for ground data.

\begin{tabular}{l|ccc}
\hline$V$ & $\langle V-I\rangle$ & $\sigma$ & error \\
\hline $15.50-16.00$ & 1.11 & 0.02 & 0.02 \\
$16.00-16.50$ & 1.08 & 0.02 & 0.03 \\
$16.50-17.00$ & 1.05 & 0.02 & 0.03 \\
$17.00-17.50$ & 1.00 & 0.03 & 0.04 \\
$17.50-18.00$ & 0.97 & 0.03 & 0.04 \\
$18.00-18.50$ & 0.94 & 0.04 & 0.05 \\
$18.50-19.00$ & 0.93 & 0.04 & 0.05 \\
\hline
\end{tabular}

Secondly, we divided this interval into bins of $0.1 \mathrm{mag}$ and computed the mean color for each bin.

The MSTO magnitude was then obtained by taking the central value of the bin for which we have the bluest mean color.

In order to build a fiducial line along the RGB for ground based data, we assumed a Gaussian color distribution and determined the mean color and dispersion, $\sigma$, in each magnitude interval. In Table 2 the bin width, the mean, the dispersion $(\sigma)$ in color and the photometric errors are listed.

Comparing the observed widths with the photometric errors, the former appear compatible with a null color dispersion along the various evolutionary phases. This fact translates into a null dispersion in metallicity (Renzini \& Fusi Pecci 1988).

\subsection{The RGB bump}

One of the most interesting features of the RGB luminosity function of galactic globular clusters is the RGB bump. It appears as a peak in the differential luminosity function and as a change in the slope of the cumulative luminosity function. From a theoretical point of view, the presence of the bump along the RGB of globular clusters was first predicted by Thomas (1967) and Iben (1968).

From an evolutionary point of view, the existence of the bump is due to the fact that during the RGB evolution, the H-burning shell crosses the chemical discontinuity left over by the convective envelope soon after the first dredgeup. The bump is located on the RGB at a luminosity which depends on the metallicity and on the age of the cluster and, in general, is not a prominent feature. In the last few years, the RGB bump has been a crossroad for several theoretical and observational investigations (see Zoccali et al. 1999 and references therein). Until few years ago, the detection of the bump was mainly hampered by the size of the available sample of RGB stars. This problem has been particularly relevant for the most metal-poor clusters, where the bump shifts toward brighter magnitudes and then less populated RGB regions. This is why we have tried to detect the bump in NGC 6101 by taking advantage of the large statistical sample of RGB stars in our CMDs. An accurate inspection of CMD of Fig. 2 (left) suggests that there is a bunch of RGB stars located at $V \simeq 16.3$ and $(V-I) \simeq 1.1$. In Fig. 3, we have plotted 

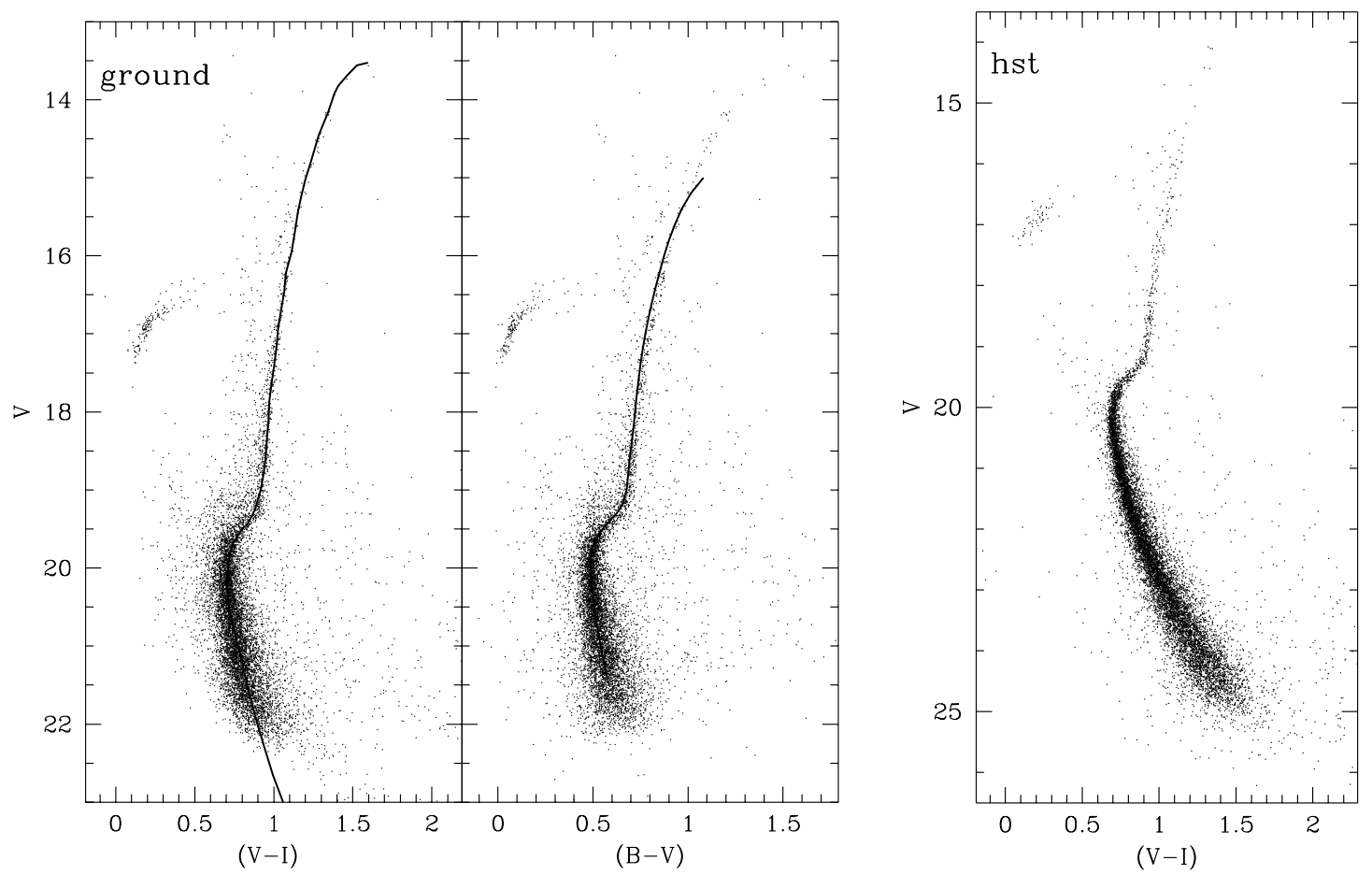

Fig. 2. Left: $(V-I),(B-V)$ Color-Magnitude Diagrams obtained by using ground data. The superimposed lines are respectivaly: the ridge line obtained by using $\operatorname{HST}(V-I) \mathrm{CMD}$; the fiducial $(B-V)$ line described in Table 2 of SDC. Right: $(V-I)$ Color-Magnitude Diagram obtained by using HST data.

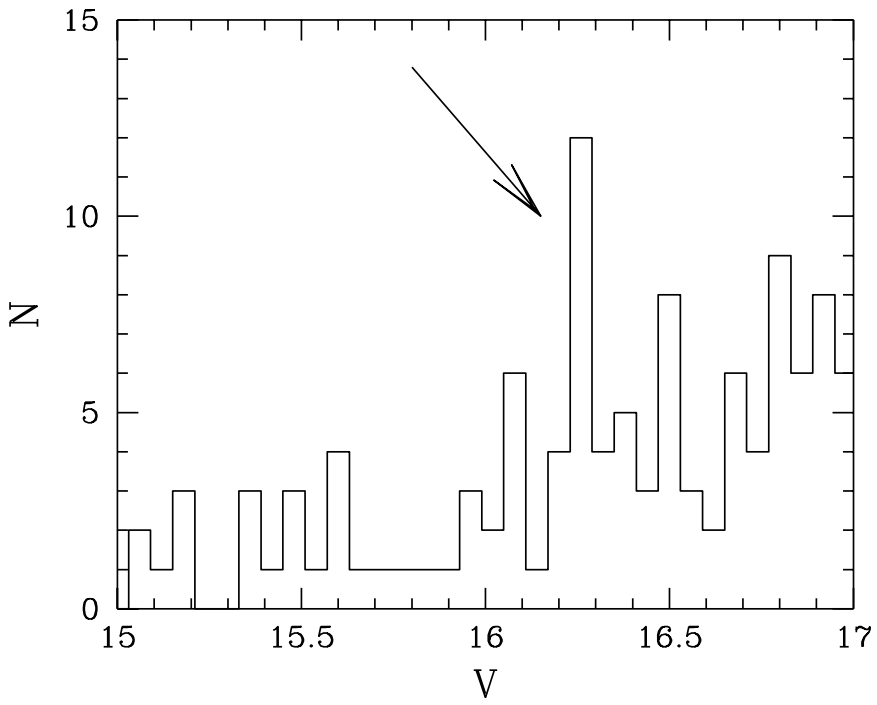

Fig. 3. Differential luminosity function for the RGB in bin of 0.06 magnitude $V$. The position of the RGB bump is marked by an arrow.

the differential luminosity function for the RGB by adopting a bin size of 0.06 mag. It is worth noticing that the peak of the bump is a rather clear feature at magnitude $V=16.26 \pm 0.03 \mathrm{mag}$.

\subsection{The horizontal branch}

Starting from Alcaino (1974), all previous photometric studies have emphasized the "normal metal-poor"

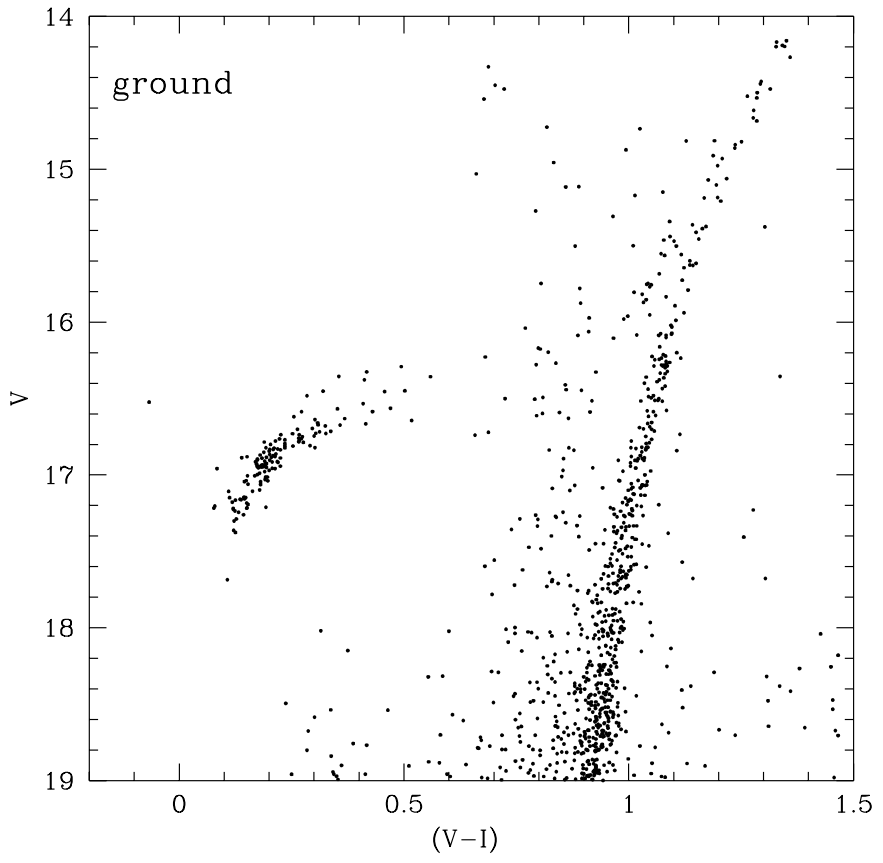

Fig. 4. Enlargement of the HB region of the $(V-I)$ CMD of NGC 6101.

morphology of the horizontal branch of NGC 6101, showing, as expected, a blue tail. In Fig. 4 an enlargement of the HB region of the CMD of NGC 6101 is shown.

We can clearly see a populated long blue tail starting from the blue edge of the RR Lyrae gap $(V-I) \simeq$ 0.3 and extending for almost 0.8 magnitudes down to 


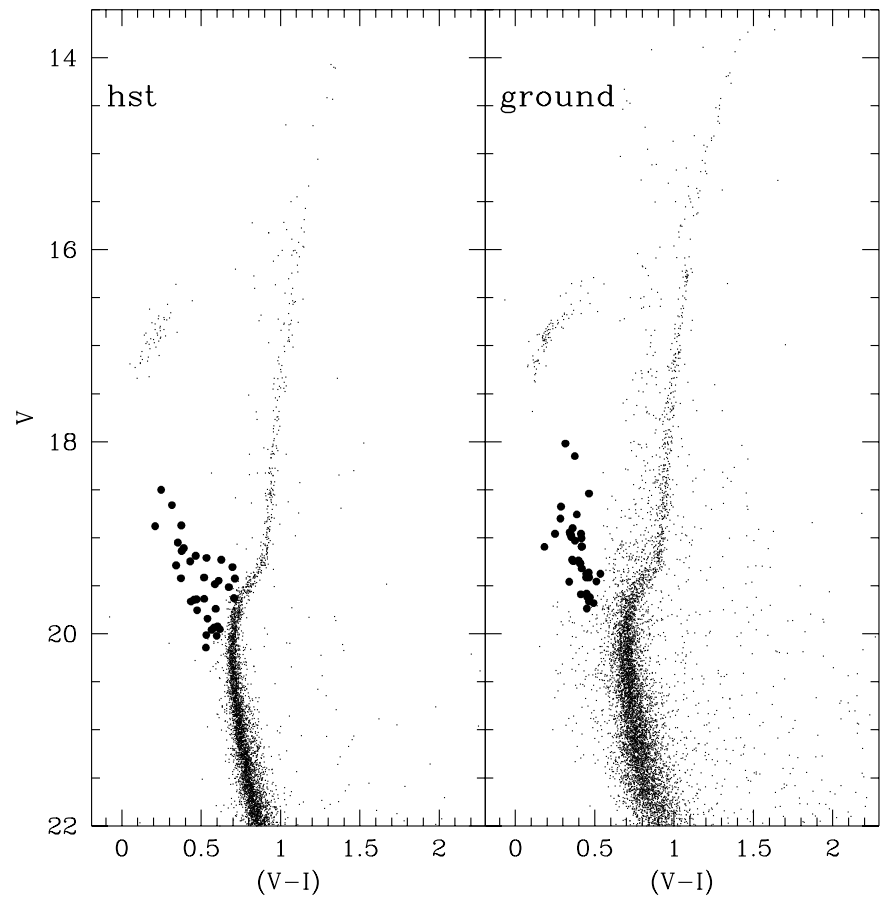

Fig. 5. Left: $(V-I)$, Color-Magnitude Diagram obtained by using HST data. Black big points represent the candidate BSS identified in the HST catalog. Right: $(V-I)$, Color-Magnitude Diagram obtained by using ground observations for stars located in the region external to HST fields. Black big points represent the candidate BSS identified in the ground catalog in this region of the sky.

$V \simeq 17.4$. There is a well visible gap in the distribution of the BHB stars starting at $V \simeq 17$. Following the methodology described in Sect. 6 we have derived for the horizontal branch $V_{\mathrm{HB}}=16.59 \pm 0.1$; this error is mainly caused by the difficulty to determine the luminosity level of such a blue HB (see also Sect. 6). All these values are in fairly good agreement with SDC and Rosenberg et al. (2000).

\subsection{Blue Stragglers}

The Blue Stragglers (BSS) are the stars located in the region betweeen the TO and the blue tail of the HB.

Several studies (e.g. Bolte et al. 1993 and Ferraro et al. 1995) suggested that the frequency of BSS should be higher in the densest clusters. In the most accepted hypothesis, BSS are the result of the merging of two MS stars. In this framework, the mass of a BSS, $m_{\mathrm{BSS}}$ should always be less than twice the mass of a TO star.

In Fig. 5 a well defined "sequence" of stars (73 BSS) bluer and brighter than the TO stars is clearly visible. We have found 46 additional BSS candidates with respect to SDC; this is probably due to the different spatial coverage of our frames, which are more central than the SDC fields.

The candidate BSS of Fig. 5 have been identified through the following procedure:

1. For the stars located in the region of the sky not included in HST fields, we selected a BSS locus on the ground ( $V$ vs. $V-I$ ) CMD and including all the objects plotted as big black points in Fig. 5 (right).

This locus has been selected as the region between the two gaps dividing the region where the candidate BSS are located from the field stars region and from the SGB, clearly visible in the figure at $(V-I \leq 0.5$, $V \leq 19.4)$ and $(V-I \leq 0.5, V \geq 19.7)$.

These candidates have been inspected on the original deep frames, and only the stars detected in all deep frames and in all bands have been classified as BSS;

2. For the sky region covered by the HST observations, we selected the BSS locus in the CMD obtained with HST (following the same procedure as in 1.) and including all the objects plotted as big black points in Fig. 5 (left). We also identified all these objects in the ground CMD by looking at the position of the objects in the frames, to check for the consistency of the sample. In order to accept an object as a candidate BSS, we compared the outputs of the deep photometries coming from the 6 different HST frames in both filters, $V$ and $I$, and for each WFPC2 camera. We accepted as candidates only the objects with at least 3 measurements in each filter and rejected the others.

The comparison among the 6 different magnitudes associated with each object allowed us to check the variability of the candidate BSS as well. We did not find any significant variability of the candidate BSS coming from HST observations.

We created a catalog of 73 BSS, 28 of which have been identified in both ground and HST catalogs; 4 have been identified only in the HST catalog; 25 have been identified on ground data-sample in the region external to HST fields and 16 BSS have been identified in common with SDC. Note that, by using HST data, we found that the star classified as the BSS number 24 by SDC (see their Table 3), is not a real BSS but a blend of two very close stars, identified as a single object from the ground.

In our analysis we also rejected 4 other objects classified as BSS from SDC (stars 3, 4, 14 and 26 in Table 3 of SDC), because they fall on the MS in our catalog.

In the CMDs of Fig. 6 the BSS candidates are reported together with: a $Z=0.0004,13$ Gyrs isochrone with a TO mass $\simeq 0.8 M_{\odot}$ and some evolutionary tracks corresponding to $1,1.1,1.2$ and $1.4 M_{\odot}$ models, bottom to top respectively.

It appears from the figure that the brighter BSS on the sample have a mass $\leq 1.4 M_{\odot}$; this means that all the BSS candidates in NGC 6101 are consistent with the hypothesis that $M_{\mathrm{BSS}} \leq 2 M_{\mathrm{TO}}$.

The radial distribution of these objects will be analyzed in Sect. 5.

\section{Completeness and luminosity function}

For HST data, incompleteness corrections were estimated as a function of the $I$ magnitude, by using the procedure based on selected real stars: the real stars were added 


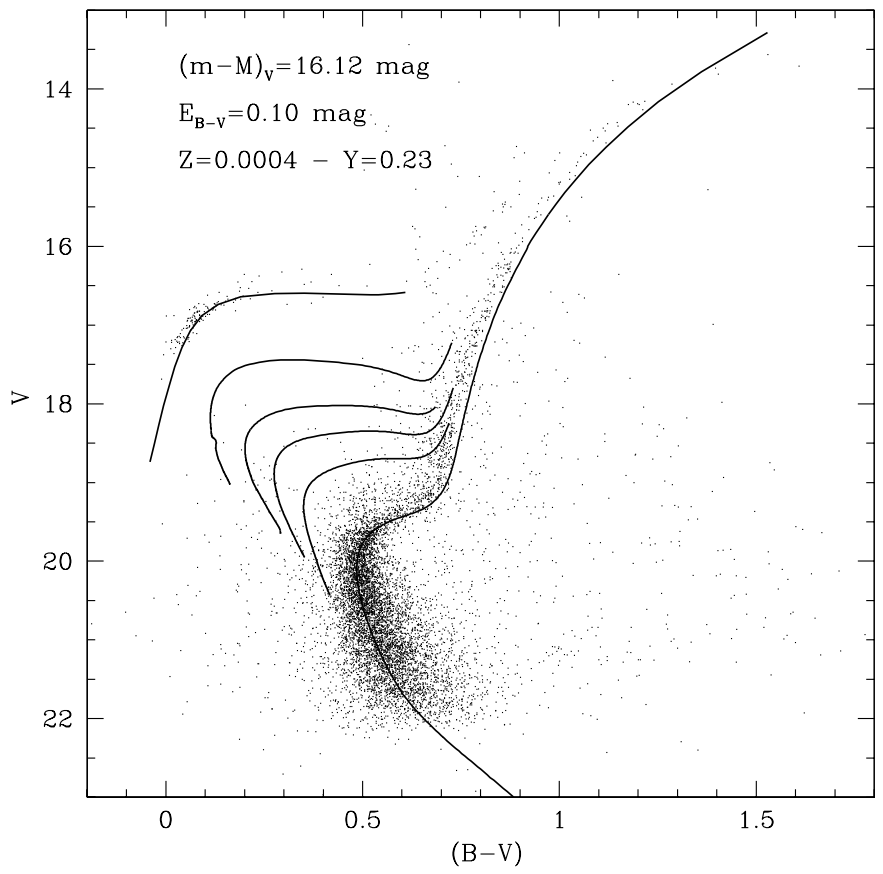

Fig. 6. Comparison between the $(V, B-V)$ Color-Magnitude Diagram obtained by using ground data and theoretical tracks corresponding at 1, 1.1, 1.2 and $1.4 M_{\odot}$. A 13 Gyrs isochrone with a TO mass of $0.8 M_{\odot}$ is also shown.

randomly to the $I$ frames used for the initial search of stellar peaks, paying attention to add a few percentage $(\leq 10 \%)$ of the total number of stars actually present in the frames, to prevent an unrealistic enhancement of the image crowding. The data reduction process described in Sect. 2.2 was then repeated from the peak detection phase to the profile fitting, thus obtaining positions and instrumental magnitudes for all the objects in the data-sample.

As a basic criterion, if a star in the output file satisfied the condition $(\Delta X, \Delta Y<1.5$ pixel, $\Delta$ mag $<0.3)$ with respect to the input star $\left(N_{\text {sim }}\right)$, it was added to the number of recovered objects $\left(N_{\text {rec }}\right)$. The ratio $N_{\text {rec }} / N_{\text {sim }}=\Phi$, the completeness factor, was derived through a minimum of 10 trials for each bin of magnitude, then calculating an average within each bin.

We built the luminosity for the three bands by dividing each CMD in 0.5 mag-wide bins, and counting the bona fide MS stars in each bin.

In particular, the cumulative luminosity functions in Fig. 7 have been obtained by counting the number of objects brighter than a fixed magnitude $I$. This figure shows a comparison between the $I$-cumulative luminosity functions obtained by using both ground-observations (for stars located in the region of the sky in common with HST-observations), and HST observations. We use the HST luminosity function to verify the completeness of ground data for the evolved stars (i.e. brighter than the TO magnitude).

The comparison between the two cumulative luminosity functions shows that ground-data can be considered $100 \%$ complete down to the magnitude of the MSTO

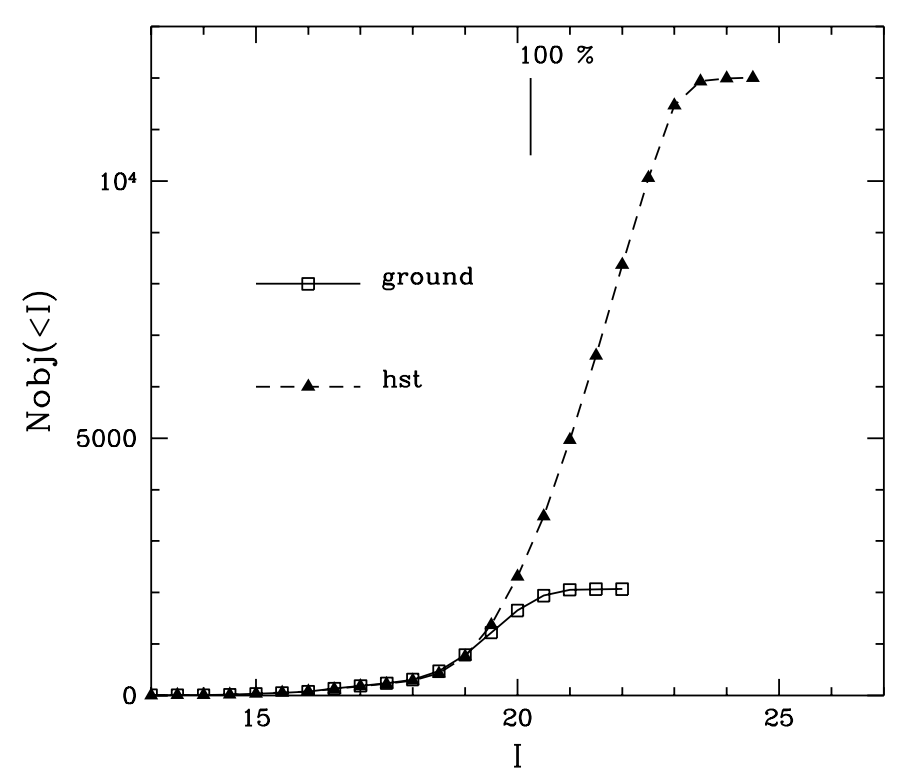

Fig. 7. Comparison between $I$-cumulative number counts of stars coming from ground observations (for the region in common with HST observations), and the $I$-cumulative number counts coming from HST observations. The percentage on the top panel is the point at which the magnitude $I$ drops down $100 \%(I \simeq 20)$.

( $I \simeq 19.35$ ), because above this magnitude the completeness of HST data is $\simeq 100 \%$.

This is a key point in our further analysis, because it allows us to use ground-observations to study the radial distributions of the evolved stars in detail.

\section{Radial distributions}

Because of the excellent seeing conditions of the ground observations, the cross-checking with HST data, and the relative looseness of NGC 6101, we are confident that we sampled almost all the stars down to $I=20$ inside 3 core radii $\left(r_{\mathrm{c}} \sim 1^{\prime}\right.$, Harris 1996). Thus, the comparison among radial distributions of stars belonging to the various evolutionary phases is not affected by incompleteness.

Figure 8 represents the stellar samples over the full area of the CCD $\left(r<230^{\prime \prime}\right)$, selected to compare the radial cumulative distributions (RCD) of the stars in different evolutionary phases. The horizontal branch has been subdivided in three distinct groups: HB bluer than the gap visible at $I \sim 17,(V-I) \sim 0.1$ (BHB), the core of He-burning stars comprised between the blue gap and the RR Lyrae gap at $(V-I) \sim 0.3(\mathrm{HB})$, and finally the supra$\mathrm{HB}$ evolved stars (EHB). In order to check the presence of a possible over-concentration of BSS, we compare - in Fig. 9 - the RCDs of BSS with those of MS, bright tip of the red giant branch (TRGB), and HB stars.

The BSS have been selected by following the procedures already described in Sect. 3. The 73 BSS (short dashed line) appear to be more concentrated than the MS stars (continuous thick line). 


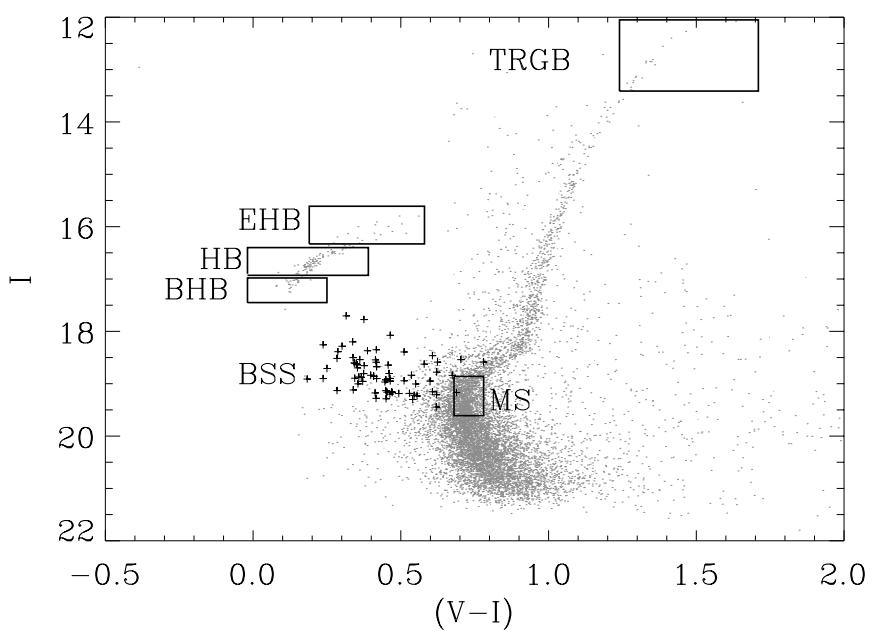

Fig. 8. $(I, V-I)$ CMD of NGC 6101 obtained by using ground data. The stellar samples adopted in order to compare their radial distributions are encircled by boxes.

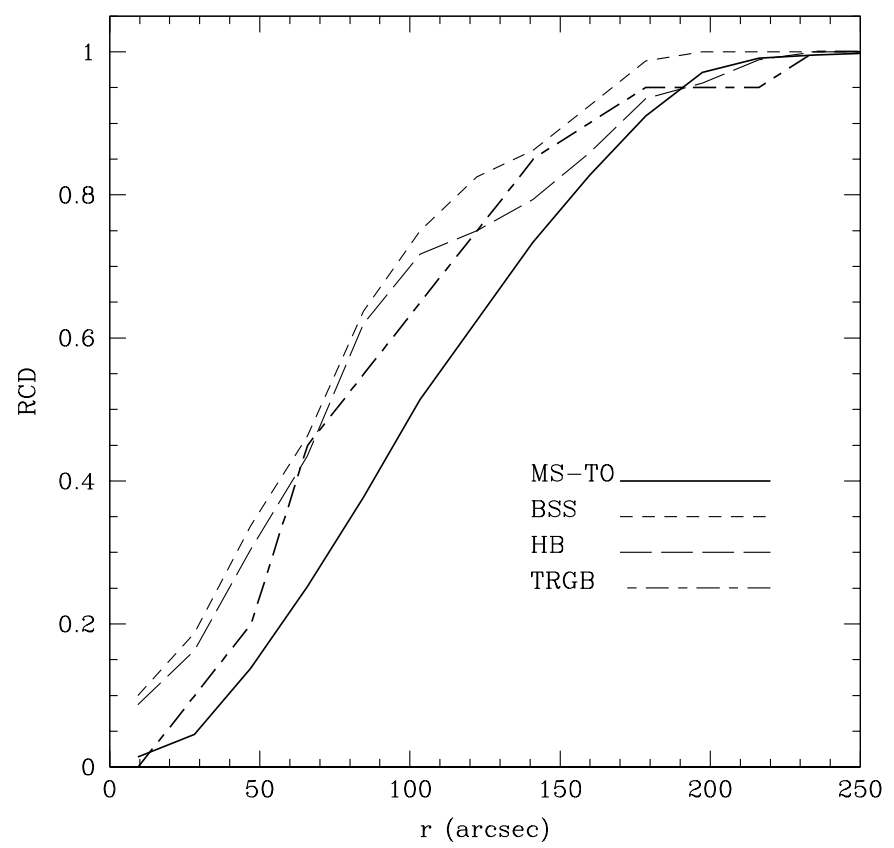

Fig. 9. The radial distribution of the MS sample (continuous thick line) is compared with those of the RGB tip (short-long dashed), HB (long dashed) and BSS (short dashed).

A two-sided Kolmogorov-Smirnov (K-S) test has been applied in order to estimate the statistical significance of the detected difference between couples of stellar types. The test yields that the BSS population is more centrally concentrated than the MS stars at the $99.9 \%$ level of significance, as well as in most galactic globulars observed at their center (Guhathakurta et al. 1998; Testa et al. 2001). This result is consistent with the hypothesis that BSS are more massive binary systems (i.e. $1-1.4 M_{\odot}$ ) than the bulk of the cluster stars.

The bright RGB stars are more concentrated than MS-TO stars. The K-S probability that the RGB stars are drawn from the same radial distribution as the faint

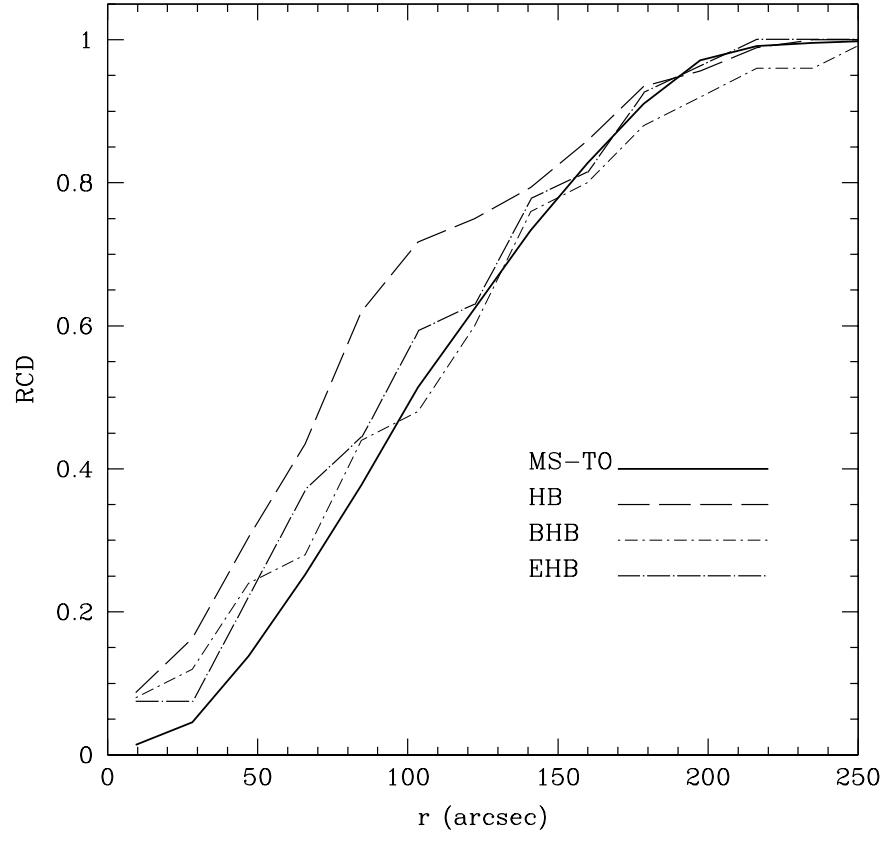

Fig. 10. Comparison between cumulative radial distributions of HB and MS-TO stars (continuous thick line). HB stars have been subdivided into three groups (see Fig. 8); HB (long dashed line) is the main body of HB stars included between the blue gap $(V-I \sim 0.2, I \sim 17)$ and the RR Lyrae gap; BHB (dotted short dashed line) represents the stars bluer than the gap; EHB (dotted long dashed line) indicates the HB stars evolving out of the central He-burning sequence.

MS-TO stars, is less than $18 \%$. However, the stellar mass at the tip of the RGB is $\sim 0.2 M_{\odot}$ lighter than TO stars, because of mass loss during the shell H-burning phase, the TRGB stars retain memory of the position inside the cluster on the basis of their initial mass. Indeed, the dynamical timescale of the two-body relaxation mechanism is much longer than the evolutionary timescale along the RGB phase.

The HB stars (long dashed line), as defined in the box of Fig. 8, are more concentrated than MS stars, following almost the same distribution of the BSS sample. K-S test yields a probability of $0.007 \%$ of equal radial distributions between $\mathrm{HB}$ and MS, and $84 \%$ between $\mathrm{HB}$ and BSS.

Figure 10 shows an intriguing feature of the HB's RCDs. The distribution of BHB stars is less concentrated than the bulk of HB objects. K-S test gives a probability of $\sim 47 \%$ of the same radial distribution. This value is even reduced if the comparison is confined inside $140^{\prime \prime}$ from the cluster center. The same result is obtained when the HB distribution is compared with that of EHB stars. EHB stars can be identified as HB stars which leave the ZAHB phase starting from the very blue side of the horizontal branch (Castellani et al. 1991). This theoretical hint is reinforced by the RCDs of both BHB and EHB. Their radial distributions have the same value at the $99.99 \%$ level of significance.This last result, together with the presence of a small gap between $\mathrm{BHB}$ and $\mathrm{HB}$, could be explained by 
adopting the suggestion of Buonanno et al. (1997): stellar collisions in the dense central environment of a cluster might be at the origin of the blue-sided population of the HB. The RCDs of BHB and EHB could be explained as a further hint of close three-body interactions in a dense environment.

Probably the ligthest stars, after a tidal-driven extra mass-loss, have been kicked off toward the periphery of the cluster. However, as a matter of fact, NGC 6101 is not a dense cluster. Its concentration is much lower than that of clusters with an extended blue HB tail (Testa et al. 2001). Hence, why does NGC 6101 show the quoted peculiar radial distribution of HB stars together with one of the highest specific frequencies of the BSS, even if it is at present a sparse cluster?

A possible answer could be found in the dynamical interactions with the disk and/or the bulge of the Galaxy. Takahashi \& Portegies Zwart (2000) have compared Fokker-Planck GC models, including an improved treatment of the tidal boundary, with $N$-body calculations. They carried out extensive calculations of the dynamical evolution of clusters over a wide range of initial conditions. They found a class of models in which, depending on the initial parameters, the tidal dragging of the low-mass stars drives the continuous decreasing of the total mass and of the concentration parameter.

Therefore, we might now be seeing the effect of the "dynamical moulding" onto a much denser cluster. In this case, the BSS specific frequency, BHB and EHB radial distributions could be the relics of the preceding dynamical evolution of NGC 6101.

\subsection{Comparison with other clusters}

A meaningful comparison of the BSS RCDs can be only made among clusters in which stars are counted in sky areas defined homogeneously. On this condition, we have compared the specific frequency $F_{\mathrm{HB}}^{\mathrm{BSS}}=N_{\mathrm{BSS}} / N_{\mathrm{HB}}$ of NGC 6101 with those of two clusters which have different values of concentration parameter, namely NGC $5897(c=$ $0.79)$ and NGC $6626(c=1.67)$ (Testa et al. 2001). In the previous formula, $N_{\mathrm{BSS}}$ is the number of BSS and $N_{\mathrm{HB}}$ is the number of HB stars in the same area. The ratio 0.58 of NGC 6101 is similar to that of NGC 5897 $\left(F_{\mathrm{HB}}^{\mathrm{BSS}}=0.48\right)$ and more than 3 times that of NGC 6626 $\left(F_{\mathrm{HB}}^{\mathrm{BSS}}=0.17\right)$. The similarity between NGC 6101 and NGC 5897 is noticeable also if we compare their RCDs. Figure 11 reports the BSS RCDs of NGC 6101 and those of the two quoted comparison clusters. All the RCDs have been normalized to the total number of BSS and plotted in units of half-mass radius $\left(r_{\mathrm{h}}\right)$. As shown in the plot, the RCD of NGC 6101 is very similar to that of NGC 5897 and much less concentrated than that of NGC 6626 .

These results suggest that NGC 6101 and NGC 5897 could have experienced similar dynamical histories. In fact, they do have almost the same structural parameters and chemical compositions.

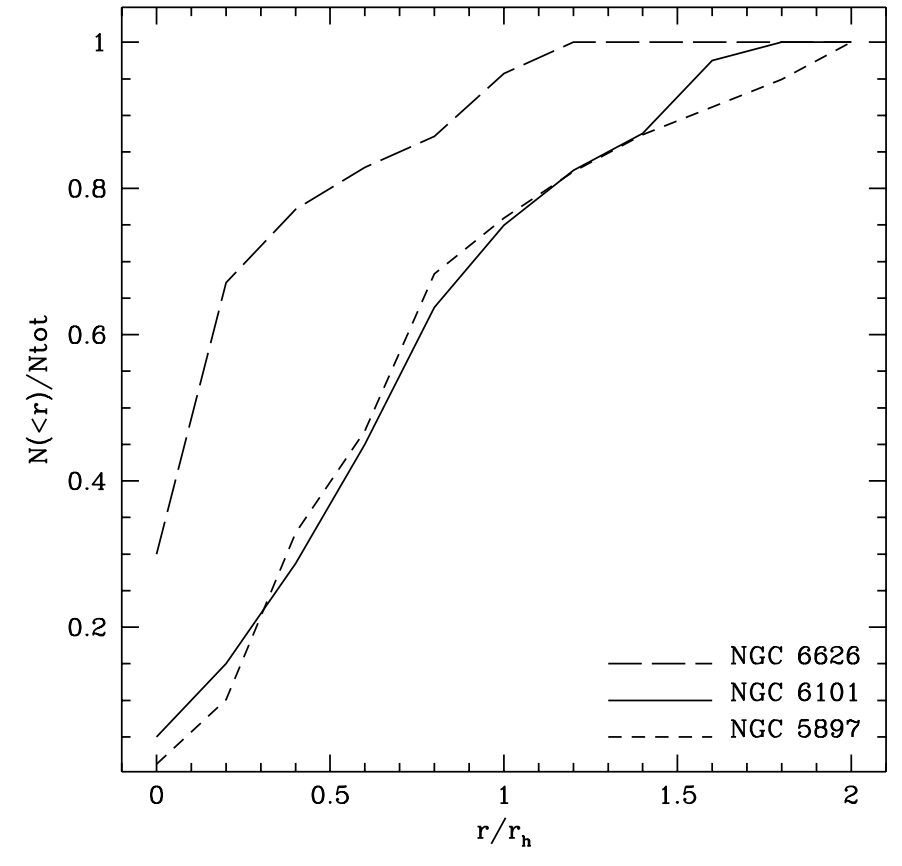

Fig. 11. BSS RCD of NGC 6101 compared with those of NGC 5897, and NGC 6626. The RCDs are normalized to the total number of BSS and are plotted in units of $r_{\mathrm{h}}$.

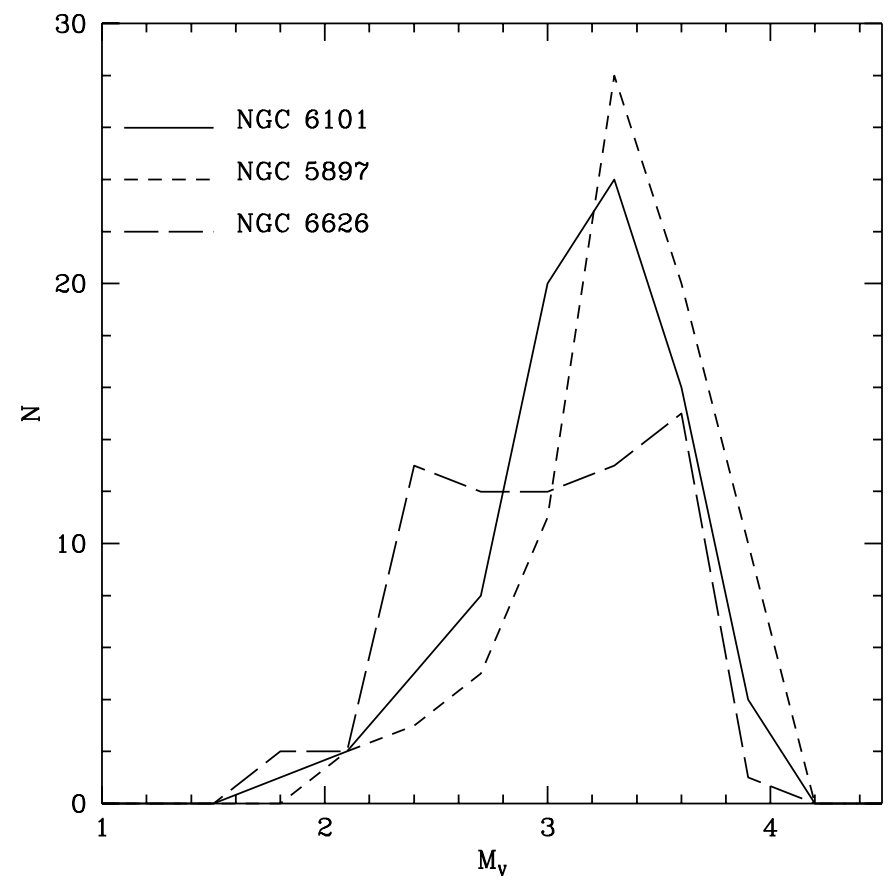

Fig. 12. BSS luminosity function in absolute $V$ magnitudes of NGC 6101 compared with those of NGC 5897, and NGC 6626.

The much more concentrated distribution of BSS in NGC 6626 might suggest a different formation mechanism with respect to the BSS of the two less concentrated clusters, NGC 6101 and NGC 5897. According to Bailyn \& Pinsonneault (1995), the shape of the LF of the BSS could be explained by invoking different formation scenarios.

Figure 12 shows the luminosity functions of the BSS in the three quoted clusters. Once again, the hypothesis of a 
substantial similarity between NGC 6101 and NGC 5897 is strenghtened. The shape of their LF is comparable, thus suggesting the same origin of their BSS population. On the contrary, the LF of NGC 6626 is almost flat with a luminous tail. This favours the hypothesis of a collisional scenario in a dense environment as the most probable BSS formation mechanism.

\section{Comparison with theory}

The high quality of the photometry allows us to perform a robust, and detailed quantitative comparison between the observational data for NGC 6101 and the theoretical predictions provided by updated stellar models. This combination is quite relevant in order to estimate the age of this cluster and its distance modulus.

We have computed a set of stellar models by using the most updated physical inputs. All the evolutionary computations presented in this paper have been performed with the Frascati RAphson Newton Evolutionary Code (FRANEC) (Chieffi et al. 1989; Castellani et al. 1997). As far as the equation of state is concerned, the updated OPAL EOS (Rogers et al. 1996) has been used. In the thermodynamical regions where the OPAL EOS is not available, it has been supplemented with the Straniero (1988) EOS. We have adopted the OPAL opacity tables (Iglesias et al. 1996) combined at low temperatures with the molecular opacities provides by Alexander \& Ferguson (1994). For a more detailed discussion on the other physical inputs adopted in the evolutionary computations, see the complete discussion in Cassisi et al. (1998, 1999). It is worth noticing that the present models account for both helium and heavy metal atomic diffusion (Castellani et al. 1997). For the calibration of the superadiabatic convection we have adopted a mixing length value of 2.0 .

The color transformations and bolometric corrections used in order to transform theoretical temperatures and luminosities in the BVI magnitudes are the ones provided by Castelli et al. (1997a, 1997b).

The metal abundance of NGC 6101 has not been well estimated yet. Until a few years ago, the only reliable determination was the estimate given by $\mathrm{ZW}([\mathrm{Fe} / \mathrm{H}]=$ $-1.81 \pm 0.15 \mathrm{dex})$. This value is in good agreement with the determination obtained by SDC by adopting a photometric metallicity indicator based upon the RGB color $([\mathrm{Fe} / \mathrm{H}]=-1.78)$. More recently, Rutledge et al. (1997), using the Ca II triplet, have provided the metallicity of a large set of galactic globular clusters by rescaling their evaluations to both the ZW and Carretta \& Gratton (1997, CG) metallicity scale. For NGC 6101, they provide a metallicity of $[\mathrm{Fe} / \mathrm{H}]=-1.95$ in the $\mathrm{ZW}$ scale and of -1.76 in the CG scale. Due to the significant difference between these estimates and taking into account the evidence that their intrinsic uncertainty is, at least, of the order of 0.2 dex (see Rutledge et al. 1997 for a discussion of this topic), we conservatively adopted two different metallicity values, $Z=0.0002$ and $Z=0.0004$, for the present

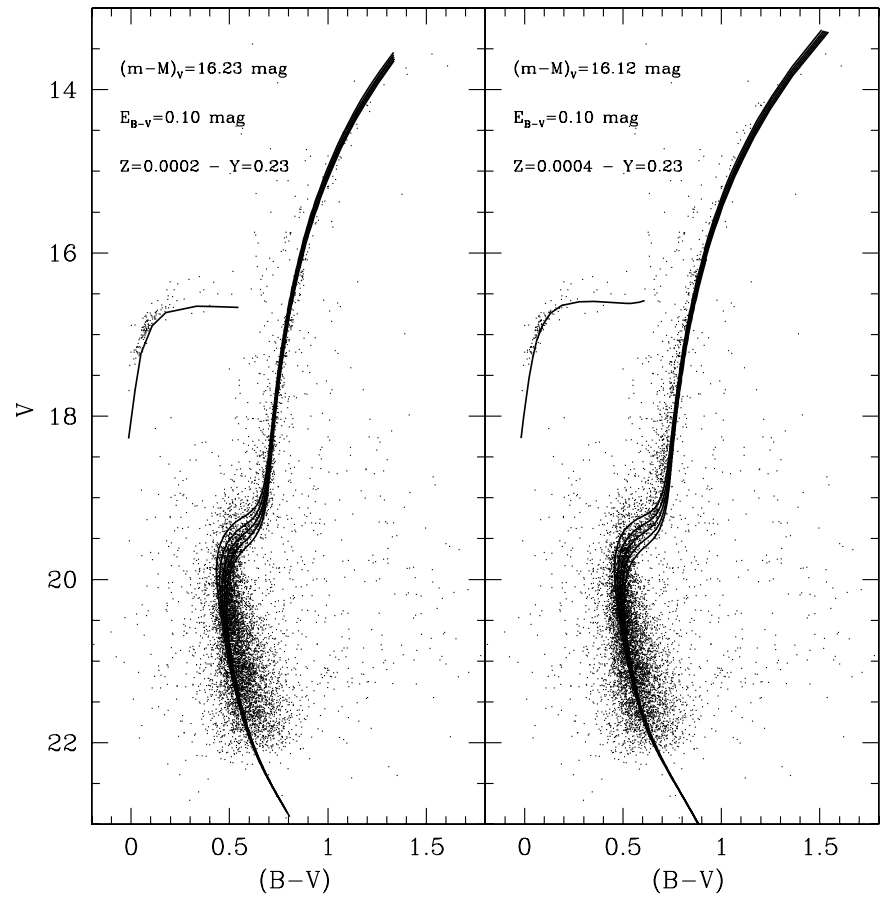

Fig. 13. Left panel: comparison between the $(V, B-V)$ CMD and isochrones for different assumptions on the cluster age: $t=10,11,12,13$ and 14 Gyrs, and for a metallicity $Z=$ 0.0002 . Right panel: the same as left panel, but for a metallicity $Z=0.0004$.

analysis in computing the stellar models. The initial He content adopted is $Y=0.23$.

In Fig. 13 (left panel), we show the comparison between the $(V, B-V)$ CMD and isochrones for different assumptions about the cluster age and a metallicity equal to $Z=0.0002$. We have also plotted the Zero Age Horizontal Branch locus corresponding to a RGB progenitor with mass equal to $0.8 M_{\odot}$.

The cluster distance modulus has been fixed so that the ZAHB locus exactly reproduces the observed lower envelope of the stellar distribution along the HB turnover. With this approach, we obtain a distance modulus $(m-$ $M)_{\mathrm{V}}=16.23$ mag. This estimate appears in good agreement with the value given by SDC, $(m-M)_{\mathrm{V}}=16.12 \mathrm{mag}$ obtained through the $M_{\mathrm{V}}(R R)-[\mathrm{Fe} / \mathrm{H}]$ relation provided by Lee et al. (1990), but it is larger by about $0.2 \mathrm{mag}$ in comparison with the value obtained by the quoted authors on the basis of isochrones fitting. In Fig. 13, we have accounted for a reddening value $E(B-V)=0.1 \mathrm{mag}$, which is in good agreement with the usually adopted estimate for NGC 6101 (see SDC). From the comparison with theoretical isochrones, one can easily find that the most suitable age for this cluster is of the order of 13 Gyrs. In the right panel of Fig. 13, we show the same comparison, but with isochrones having metallicity equal to $Z=0.0004$. In this case, we derive a distance modulus equal to 16.12 , in much better agreement with the estimate derived by SDC by using the isochrone fitting approach. For this choice the cluster age is of the order of 12 Gyrs. 


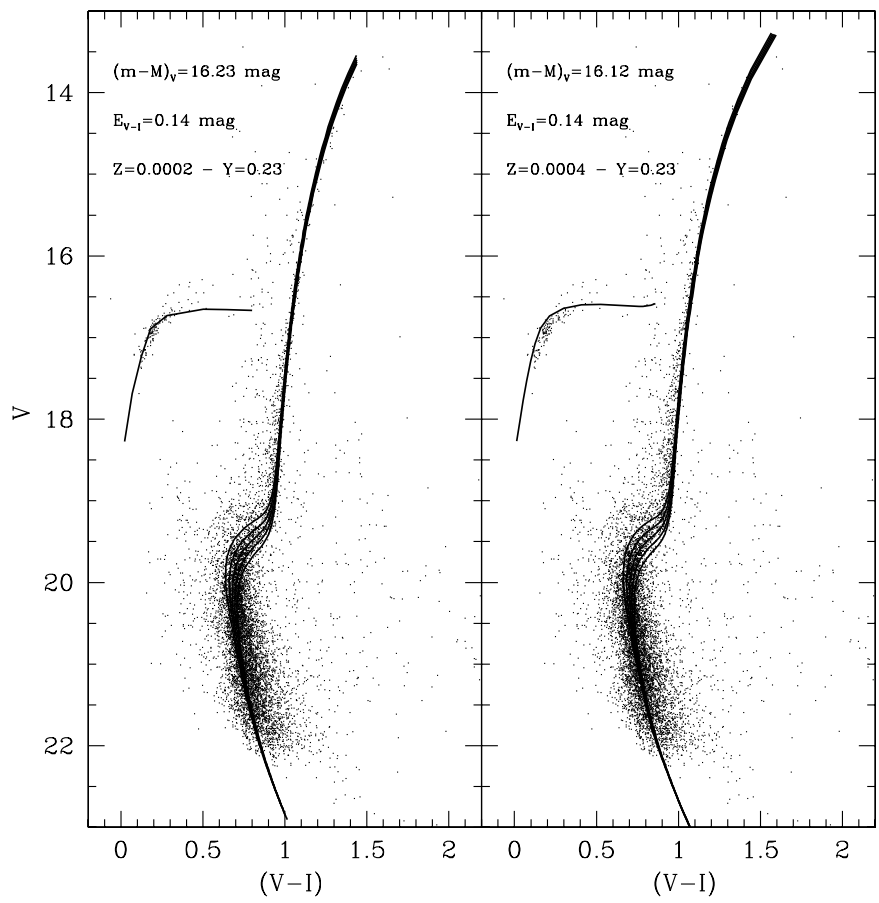

Fig. 14. Left panel: comparison between the $(V, V-I)$ CMD and isochrones for different assumptions on the cluster age: $t=10,11,12,13$ and 14 Gyrs, and for a metallicity $Z=$ 0.0002. Right panel: the same as left panel, but for a metallicity $Z=0.0004$.

Figure 14, reports the same comparison for the $(V$, $V-I)$ CMD.

We have used the same distance modulus and a reddening $E(V-I)=0.14 \mathrm{mag}$ accounting for the relation $E(V-I)=1.36 \times E(B-V)$ (Taylor 1986). It is worth noticing that, also on this observational plane, for the quoted choice of distance modulus and reddening, a good agreement exists between theoretical predictions and observational data for a cluster age equal to $\approx 13$ Gyrs. However, it is also interesting that, for the adopted distance modulus, the ZAHB locus cannot finely match the lower envelope of the observed HB distribution. This is probably due to a drawback in the adopted color-effective temperature and bolometric corrections scales (see, for instance, Weiss \& Salaris 1999). The same discrepancy is evident in the right panel of Fig. 13, where we show the theoretical predictions for a metallicity equal to $Z=0.0004$.

\section{1. "The bump"}

Since the work by Fusi Pecci et al. (1990), it is clear that a meaningful comparison between theory and observation concerning the bump location in the luminosity function can be performed by using the parameter $\Delta V_{\mathrm{HB}}^{\text {bump }}-$ commonly defined as the difference in magnitude between the RGB bump and the horizontal branch stars located within the RR Lyrae instability strip. However, the determination of the ZAHB magnitude $V_{\mathrm{ZAHB}}$ in clusters with a blue HB morphology, as in NGC 6101, is a moot point

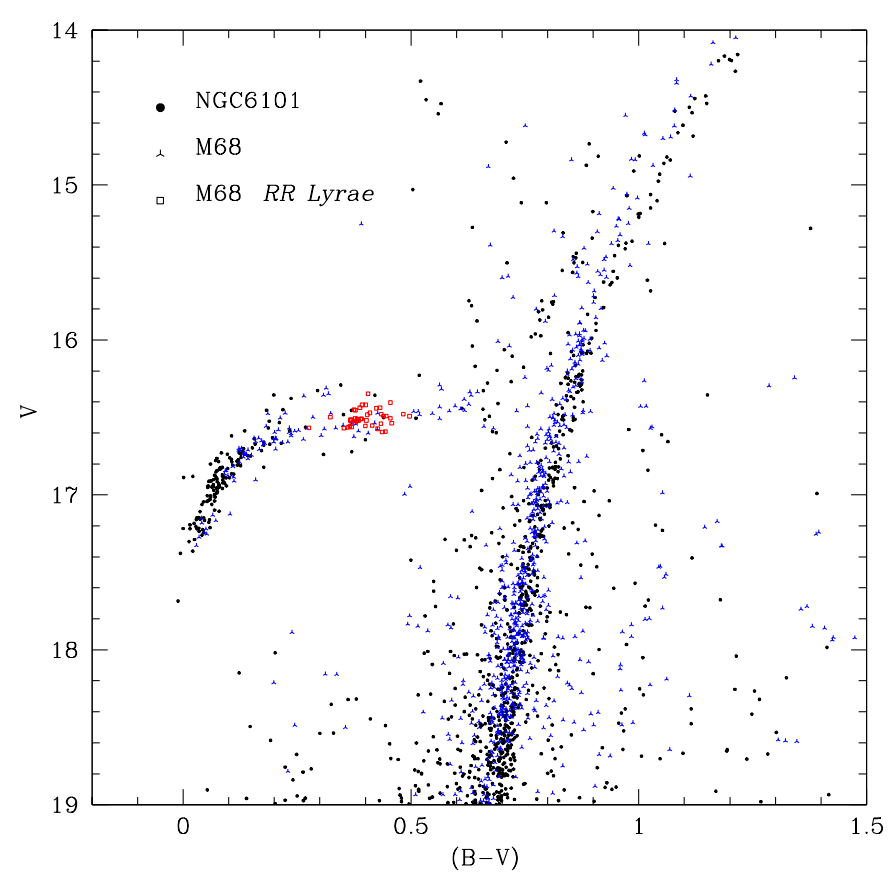

Fig. 15. Comparison between the BV-CMDs of NGC 6101 and M68 (see text for more details).

(see Cassisi \& Salaris 1997 for more details). In order to overcome this problem, we have used the same approach adopted by Cassisi \& Salaris (1997). We have chosen from the literature a cluster with metallicity similar to NGC 6101, with accurate photometry, a large number of RR Lyrae variables and a well-defined blue HB. The cluster M 68 fulfils these conditions (Walker 1994). Hence, we have shifted the CMD of this cluster in color and magnitude in order to match both the RGB and the blue HB tail of NGC 6101. The requested shift in magnitude is equal to $+0.87 \mathrm{mag}$ (see Fig. 15).

By adding this quantity to the estimated mean magnitude of the RR Lyrae in M68, $<V_{\mathrm{RR}}>=15.64 \pm$ $0.01 \mathrm{mag}$ (Walker 1994), and by applying the correction between the ZAHB luminosity level and the mean magnitude of RR Lyrae (Cassisi \& Salaris 1997), we have estimated a ZAHB magnitude within the instability strip for NGC 6101 of $V_{\mathrm{ZAHB}}=16.59$ mag. With this approach, we adopt an uncertainty on this quantity of the order of $0.10 \mathrm{mag}$. Therefore, the estimated value of $\Delta V_{\mathrm{HB}}^{\mathrm{bump}}$ for NGC 6101 is equal to $-0.33 \pm 0.10$ mag. In Fig. 16, we show the comparison between observational measurements of the parameter $\Delta V_{\mathrm{HB}}^{\text {bump }}$ (Zoccali et al. 1999), including our estimate for NGC 6101, and theoretical predictions of this quantity.

Accounting for the still large uncertainty affecting the globular cluster metallicity scale, we have adopted both the ZW and the CG scales. In addition, we have assumed that $\alpha$-elements are enhanced in all clusters (see Zoccali et al. 1999, for more details). In order to show the effect of the cluster age on the RGB bump magnitude, we have plotted relations corresponding to three different ages. We also show the theoretical relation for an age 


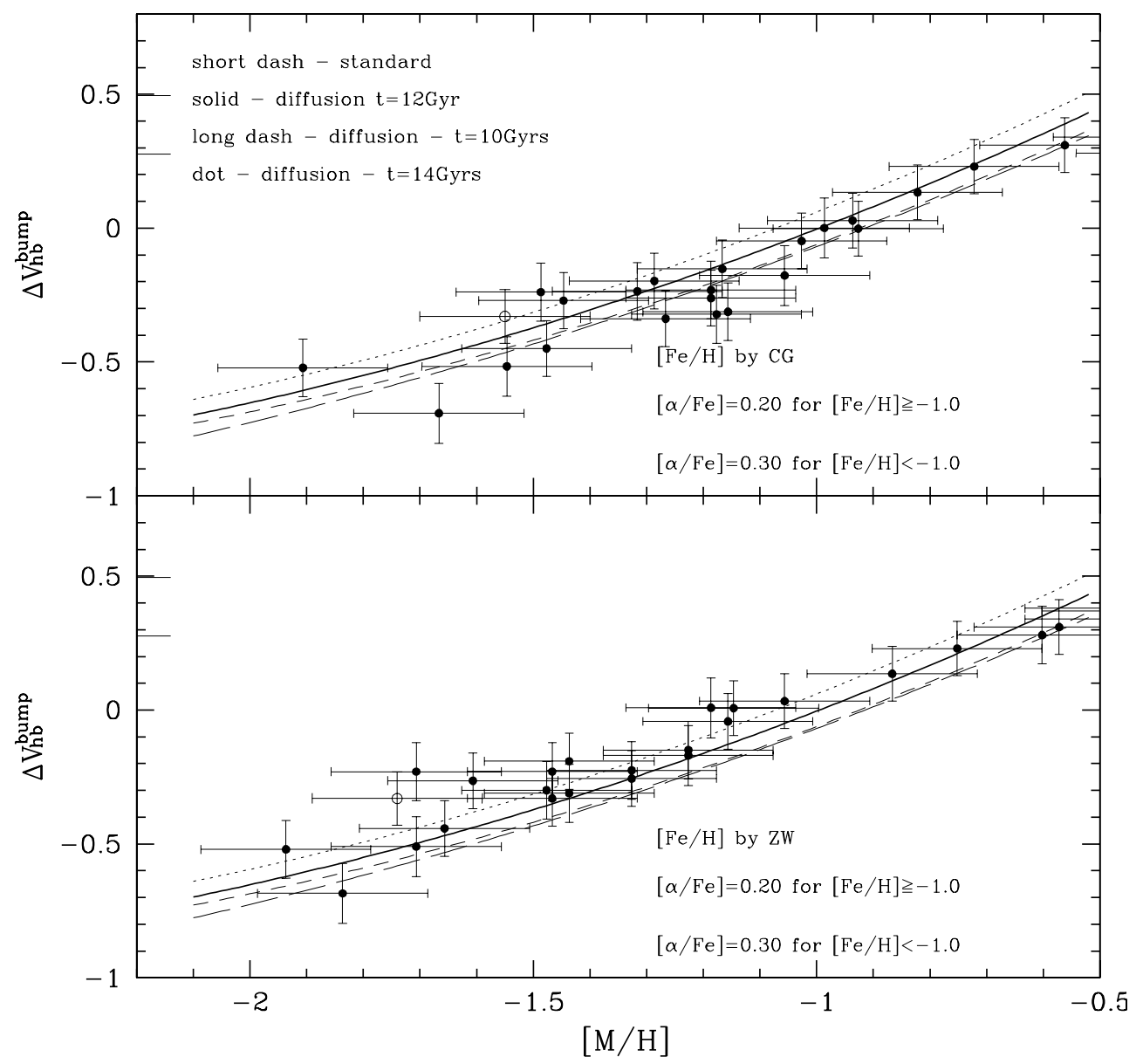

Fig. 16. Upper panel: comparison between observational measurements of the parameter $\Delta V_{\mathrm{HB}}^{\text {bump }}$ as given by Zoccali et al. (1999), including our estimate for NGC 6101 (open circle), and theoretical predictions on this quantity, by adopting the metallicity scale provided by CG. Lower panel: as in the upper panel but adopting the metallicity scale of ZW.

equal to 12 Gyr as provided by canonical stellar models, i.e. not accounting for atomic diffusion. It is worth noting that the location of NGC 6101 appears in good agreement with theoretical predictions. However, the agreement is even better if we adopt the iron abundance suggested by ZW, which is significantly lower than the one provided by Rutledge et al. (1997) on the CG scale. This result, in agreement with previous investigations performed by Cassisi \& Salaris (1997), Zoccali et al. (1999) and Alves \& Sarajedini (1999), shows that the observational evidence on the difference in magnitude between the RGB bump and HB, also in metal-poor clusters, can be well reproduced within the current theoretical framework.

\section{Summary}

We have presented a new and complete CCD photometry for $\simeq 20000$ stars from the very center out to $200^{\prime \prime}$ inside the galactic halo globular cluster NGC 6101. The new photometry is obtained from ground based and HST datasets. The photometric catalogs have been used to build $B$ vs. $(B-V)$ and $V$ vs. $(V-I)$ CMDs. The single evolutionary phases have been analysed in detail. We have also performed an extensive comparison of our data with the predictions of the stellar models.

73 BSS stars have been identified inside a radius of $\simeq 200^{\prime \prime}$. They do not show any sign of variability. We have investigated the radial distribution of the BSS. The BSS are more concentrated than MS-TO and have a spatial distribution surprisingly similar to the evolved supra-HB stars (EHB). Comparing the BSS content of NGC 6101 with another similar GGC (NGC 5897) we found that LFs and radial distributions are almost identical. Therefore, we argued that the mechanism at the origin of the formation of these objects inside the two clusters should be the same. The large statistical sample of stars in our CMDs has allowed us to obtain a precise detection of the RGB bump in this metal-poor cluster. By accounting also for the $\Delta V_{\mathrm{HB}}^{\text {bump }}$ estimates available in the literature for a large database of galactic globular clusters, we have performed a comparison between observational evidence and theoretical prescriptions for the luminosity difference between the RGB bump and the HB at the level of the instability strip of the RR Lyrae variables. As a result, we have found a good agreement - within current uncertainties - between theory and observations. 
Acknowledgements. We warmly thank G. Piotto for kindly sending us his data for NGC 6101 and G. Giobbi for careful reading of the manuscript. This work has been supported by MURST/Cofin1999 under the project: "Effect of the dynamics on the canonical and exotic stellar distributions inside galactic globular clusters". One of us (S.C.) was supported by MURST/Cofin2000 under the project: "Stellar observables of cosmological relevance".

\section{References}

Alcaino, G. 1974, A\&AS, 18, 9

Alexander, D. R., \& Ferguson, J. W. 1994, ApJ, 437, 879

Alves, D. R., \& Sarajedini, A. 1999, ApJ, 511, 225

Bailyn, C. D., \& Pinsonneault, M. H. 1995, ApJ, 439, 705

Bolte, M., Hesser, J. E., \& Stetson, P. B. 1993, ApJ, 408, L89

Buonanno, R., \& Iannicola, G. 1989, PASP, 101, 294

Buonanno, R., Corsi, C. E., Bellazzini, M., Ferraro, F. R., \& Fusi Pecci, F. 1997, AJ, 113, 706

Carretta, E., \& Gratton, R. G. 1997, A\&A, 121, 95 (CG)

Cassisi, S., \& Salaris, M. 1997, MNRAS, 285, 593

Cassisi, S., Castellani, V., Degl'Innocenti, S., \& Weiss, A. 1998, A\&AS, 129, 267

Cassisi, S., Castellani, V., Degl'Innocenti, S., Salaris, M., \& Weiss, A. 1999, A\&AS, 129, 267

Castellani, V., Chieffi, A., \& Pulone, L. 1991, ApJS, 76, 911

Castellani, V., Ciacio, F., Degl'Innocenti, S., \& Fiorentini, G. 1997, A\&A, 322, 801

Castelli, F., Gratton, R. G., \& Kurucz, R. L. 1997a, A\&A, 318, 841

Castelli, F., Gratton, R. G., \& Kurucz, R. L. 1997b, A\&A, 294, 80; A\&A, 324, 432

Chieffi, A., \& Straniero, O. 1989, ApJS, 71, 47

Djorgovski, S. 1993, in Structure and Dynamics of Globular Clusters, ed. S. G. Djorgovski, G. Meylan, ASP Conf. Ser., 50,373
Ferraro, F. R., Fusi Pecci, F., \& Bellazzini, M. 1995

Fusi Pecci, F., Ferraro, F. R., Crocker, D. A., Rood, R. T., \& Buonanno, R. 1990, A\&A, 238, 95

Guhathakurta, P., Zodiac, T. W., Yanni, B., Schneider, D. P., \& Bahcall, J. N. 1998, AJ, 116, 1757

Harris, W. E. 1996, ApJ, 112, 148

Holtzman, J. A., Burrows, C. J., Casertano, S., et al. 1995, PASP, 107, 1065

Iben, I. Jr. 1968, ApJ, 154, 581

Iglesias, C. A., \& Rogers, F. J. 1996, ApJ, 464, 943

Landolt, A. U 1992, ApJ, 104, 340

Lee, Y.-W., Demarque, P., \& Zinn, R. 1990, ApJ, 350, 155

Pryor, C., Smith, G. H., \& McClure, R. D. 1986, AJ, 92, 138

Renzini, A., \& Fusi Pecci, F. 1988, ARA\&A, 26, 199

Rogers, F. J., Swenson, F. J., \& Iglesias, C. A. 1996, ApJ, 456, 902

Rosenberg, A., Piotto, G., Saviane, I., \& Aparicio, A. 2000, A\&AS, 144, 5

Rutledge, G. A., Hesser, J. E., \& Stetson, P. B. 1997, PASP, 109, 907

Sarajedini, A., \& Da Costa, G. S. 1991, AJ, 102(2), 628 (SDC)

Stetson, P. B. 1987, PASP, 99, 191

Stetson, P. B. 1992, User's Manual for DAOPHOT-II

Straniero, O. 1988, A\&AS, 76, 157

Takahashi, K., \& Portegies Zwart, S. F. 2000, ApJ, 535, 759

Taylor, B. J. 1986, ApJS, 60, 577

Testa, V., Corsi, C. E., Andreuzzi, G., Iannicola, G., Marconi, G., Piersimoni, A., \& Buonanno, R. 2001, AJ, 121, 916

Thomas, H.-C. 1967, Z. Astrophys., 67, 420

Walker, A. R. 1994, AJ, 108, 555

Weiss, A., \& Salaris, M. 1999, A\&A, 346, 897

Zinn, R., \& West, M. J. 1984, ApJS, 55, 45 (ZW)

Zoccali, M., Cassisi, S., Piotto, G., Bono, G., \& Salaris, M. 1999, ApJ, 518, L49 